\title{
Evolution of magnetic helicity in the course of kinetic magnetic reconnection
}

\author{
T. Wiegelmann ${ }^{1,2}$ and J. Büchner ${ }^{1}$ \\ ${ }^{1}$ Max-Planck-Institut für Aeronomie, Max-Planck-Str. 2, 37191 Katlenburg-Lindau, Germany \\ ${ }^{2}$ School of Mathematics and Statistics, University of St. Andrews, St. Andrews, KY16 9SS, United Kingdom
}

Received: 20 September 2000 - Revised: 17 January 2001 - Accepted: 5 March 2001

\begin{abstract}
We investigate the evolution of magnetic helicity density in the course of 2D and 3D kinetic magnetic reconnection through thin current sheets. In 2D, the helicity density near a reconnection $\mathrm{X}$-line becomes purely quadrupolar structured, while in 3D, an additional dipolar structure occurs. This dipolar structure is related to kinetic current instabilities and becomes dominant for spontaneous 3D reconnection, in accordance with the dominating current instabilities. The 2D simulations have been carried out with a newly developed Vlasov-code and the 3D simulations with the particlein-cell code GISMO.
\end{abstract}

\section{Introduction}

Magnetic reconnection is an important process in space plasmas. Examples in the solar system where magnetic reconnection is assumed to play an important role are coronal mass ejections (e.g. Low, 1994; Schwenn et al., 1997; Wiegelmann et al., 2000) and geo-magnetic substorms (e.g. Birn, 1980). An important constraint is the magnetic helicity. Most solar system plasmas as the solar corona or the Earth's magnetotail are nearly ideal, and the plasma is frozen in the magnetic field. In a strictly ideal plasma, changes in topology are not possible and thus, magnetic reconnection cannot occur; the magnetic helicity is conserved exactly (e.g. Woltjer, 1960). A strictly ideal plasma, however, does not exist in nature and thus, magnetic reconnection is possible, in principle. It has been conjectured by Taylor (1974) that the magnetic helicity is still approximately conserved during relaxation processes involving magnetic reconnection. Later, Berger (1984) proved that the total helicity is decreasing slower than the magnetic energy. Under very special conditions, with strictly antiparallel magnetic fields, the magnetic helicity is conserved exactly under magnetic reconnection. As pointed out by Hornig (1999a), such configurations fulfill $\boldsymbol{E} \cdot \boldsymbol{B}=0$ and consequently, the parallel electric field vanishes. This

Correspondence to: T. Wiegelmann (tw@ mcs.st-and.ac.uk) condition is fulfilled in the 2D concept of magnetic merging (Vasyliūnas, 1975). Reconnection (or magnetic merging) was described there as a plasma flow across a separatrix separating regions of different magnetic topology. This implies an electric field perpendicular to the reconnection plane and parallel to the separator, and a localized violation of the ideal Ohm's law. Axford (1984) described magnetic reconnection as a localized breakdown of the frozenin field condition and the resulting changes of connection. The definition given by Vasyliūnas has the drawback of becoming structural unstable during any slight variation of the system. A more general definition of magnetic reconnection was given by Schindler et al. (1988) and Hesse and Schindler (1988). Within this concept of the so-called general magnetic reconnection, magnetic merging is only a special case with $\boldsymbol{B}=0$ in the reconnection region, known as the zero$B$ reconnection. The authors argued that in the generic 3D case, the reconnection region contains a finite magnetic field and parallel electric fields. It was also shown that a nonnegligible parallel electric field in the reconnection region has global effects. Hence, the total magnetic helicity may change. Hornig (1999b) confirmed this result in a covariant formulation, showing that for vanishing $\boldsymbol{E} \cdot \boldsymbol{B}$, the helicity is frozen in a virtual fluid flow of stagnation type. For $\boldsymbol{E} \cdot \boldsymbol{B} \neq 0$, the helicity is still approximately conserved if the non ideal reconnection zone is small, as compared to the length scale of the magnetic structure. In general, however, a non vanishing $\boldsymbol{E} \cdot \boldsymbol{B}$ may be a source for helicity production (Hornig and Rastätter, 1997).

These investigations, which were all carried out in the framework of magnetohydrodynamics, require a non ideal region, e.g. a resistivity in the reconnection region. The nature of such a resistivity cannot be calculated within MHD and ad hoc assumptions are necessary to prescribe the transport coefficients (e.g. resistivity). The concept of general magnetic reconnection by Schindler et al. (1988) assumes that a finite magnetic field component should be present in the generic case of 3D magnetic reconnection. It does not, however, identify the source of such a component within a spontaneous 
reconnection process. The cause of a non ideal behaviour in space plasma in localized regions has been assumed to be anomalous resistivity in thin current sheets. The formation of these thin current sheets can be understood within the framework of sequences of ideal MHD equlibria (e.g. Schindler and Birn, 1993; Parker, 1994; Wiegelmann and Schindler, 1995). Resistive MHD computations (e.g. Biskamp and Welter, 1980; Matthaeus and Lamkin, 1985, 1986) show the formation of current sheets as a consequence of turbulent magnetic reconnection. The further development of these thin current sheets cannot be investigated within the framework of magnetohydrodynamics since their sheet widths become comparable with the ion gyro scale and thus, kinetic effects have to be considered.

Consequently, the investigation of kinetic effects is necessary to overcome several limitations of MHD, e.g. the necessity of ad hoc assumptions for a resistivity profile, the limitations of time and length scales compared with kinetic scales, the lack of explanation for possible sources of finite magnetic field components in the reconnection zone, and the impossibility of investigating the structure of the reconnection zone. As pointed out by Winglee (1991), the generation of a magnetic field component in the current direction $\left(B_{y}\right)$ is a pure particle effect, due to the different motion of electrons and ions, as predicted by Terasawa (1983). These 2D investigations can be considered as a first step towards a full understanding of kinetic magnetic reconnection. Later, several authors pointed out the intrinsic 3D structure of kinetic magnetic reconnection (Büchner and Kuska, 1996; Pritchett et al., 1996; Zhu and Winglee, 1996; Büchner, 1999). A main difference between 2D and 3D configurations is, that in 3D configurations, additional instabilities can occur in the current direction (which is assumed to be invariant in 2D), leading to drift current instabilities (e.g. Büchner and Kuska, 1999) and these drift current instabilities couple with reconnection (e.g. Büchner, 1999; Wiegelmann and Büchner, 2000).

Here we investigate the consequences of kinetic effects on the helicity evolution in the course of reconnection. The paper is organised as follows. In Sect. 2 we outline our simulation approach and in Sect. 3, we briefly describe the Vlasovcode which has been used for the 2D investigations. Section 4 contains the results of our 2D and 3D numerical simulations. We summarize our work in Sect. 5 and give an outlook to future work.

\section{Simulation approaches}

First, it is necessary to determine the initial state of the simulation model. The basic equation is the Vlasov equation of collisionless plasmas. Stationary solutions of the Vlasov equation can be found with the help of constants of motion under some constraints. If one assumes a $2 \mathrm{D}$ configuration in space $(x, z), \partial / \partial y=0$, with the current having only a component in the invariant direction, $j_{y}$, one finds that the Hamilton function $H_{j}=\left(M_{j} / 2\right) v_{j}^{2}+q_{j} \phi$ and the generalized mo- mentum $p_{y, j}=M_{j} v_{y, j}+q_{j} A_{y}$ are constants of motion. Assuming a drifting Maxwell distribution and quasineutrality, the stationary Vlasov equation can be solved with the Grad-Shafranov-equation $-\Delta A_{y}=j_{y}\left(A_{y}\right)$. This leads us to stationary state distribution functions in the form

$f_{j}=\rho(x, z) \exp \left(-\frac{M_{i}}{2 T_{i}}\left(v_{x}^{2}+\left[v_{y}-u_{\mathrm{dyj}}\right]^{2}+v_{z}^{2}\right)\right)$

where $\rho(x, z)$ is the density profile and $u_{\text {dyj }}$ the drift velocities. A well known current sheet solution is the Harris sheet profile (Harris, 1962) which has an additional invariance in $x$ :

$\rho(z)=\frac{1}{\cosh ^{2}(z)}$

where $x, z$ are normalized by the sheet half width $L_{z}$. Configurations with a modified Harris sheet profile in $z$, but slight variations in $x$, are also solutions of the Grad-Shafranov equation (Schindler, 1972). We will use a configuration in the form:

$\rho(x, z)=\left[1-\epsilon_{1} \cos \left(2 \pi \epsilon_{2} x / L\right)\right] \frac{1}{\cosh ^{2}(z)}$

where $\epsilon_{1} \ll 1$ for a smooth variation, and $L$ is the simulation box length in $x$. With $-L / 2<x<L / 2$ and $x=0$ in the center of the box, $\epsilon_{2}=1$ will lead to a X-point in the center of the box, and $\epsilon_{2}=2$ to a sheet with X-point at $x=0$, and two O-points at $x= \pm L / 4$. With the boundary condition $A_{i}\left(z_{\min }\right)=A_{i}\left(z_{\max }\right)=0.0$, we get the flux function for the modified Harris sheet

$$
\begin{aligned}
A_{y}(x, z)= & \sqrt{\left[1-\epsilon_{1} \cos \left(2 \pi \epsilon_{2} x / L\right)\right]} \\
& \cdot\left(\ln \cosh \left(z_{\max }\right)-\ln \cosh (z)\right)
\end{aligned}
$$

The advantage of a modified Harris sheet solution (2) is that it prescribes the location of $\mathrm{X}$ - and O-points, which is useful for diagnostic purposes. Furthermore, the initial perturbation of the Harris sheet reduces the reconnection time by a factor of about 5 .

We investigate four different cases:

1. $2 \mathrm{D}$ reconnection $(\partial / \partial y=0)$ with a modified Harris sheet, according to (2) with an X-line at the center;

2. $3 \mathrm{D}$ reconnection of a Harris sheet with a $2 \mathrm{D}$ magnetic field perturbation;

3. 3D reconnection of a Harris sheet with a 3D magnetic field perturbation;

4. Spontaneous 3D reconnection of an unperturbed Harris sheet.

The pure 2D simulations are carried out with a newly developed Vlasov-code, which is briefly described in Sect. 3 . We also provide the simulation parameters for case 1 . The 3D simulations (cases 2-4) are carried out with the particle in cell code GISMO, which has been described in detail by 
Table 1. Simulation parameters

\begin{tabular}{|l|l|l|l|l|r|r|l|}
\hline & code & $\operatorname{dim}$ & $m_{i} / m_{e}$ & pert & cpu & memory & grid \\
\hline \hline 1. & Vlasov & 2D & 16 & 2D & 8h & $64 \mathrm{MB}$ & $40^{*} 20$ \\
\hline 2. & PIC & 3D & 25 & 2D & 6d & $1 \mathrm{~GB}$ & $64^{3}$ \\
\hline 3. & PIC & 3D & 25 & 3D & 6d & $1 \mathrm{~GB}$ & $64^{3}$ \\
\hline 4. & PIC & 3D & 25 & - & 21d & $1 \mathrm{~GB}$ & $64^{3}$ \\
\hline
\end{tabular}

Kuska and Büchner (1999). In the following table, we provide information about the codes used (Vlasov-code or PICcode), the dimension of the simulation box, the mass ratio, the type of perturbation, the required CPU-time (on a workstation) and the required memory.

In cases 2-4 we use the following parameter set: a grid of $64 \times 64 \times 64$ grid points and 2 million particles. The box length corresponds to $12 L_{z}$ in each direction, where $L_{z}$ is the sheet half width. We use a reduced mass ratio of ions and electrons $M_{i} / M_{e}=25$, and a temperature ratio $T_{i} / T_{e}=5$. We resolve the ion Debye length by 1.5 grid points and the electron Debye length by 0.7 grid points. We resolve a full electron gyro period by 18 integration time steps and a full ion gyro period by 450 time steps. Thus one ion gyro time $\Omega_{c i}^{-1}$ is resolved by 72 time steps. The Harris sheet half width has the same order of magnitude as the ion gyro radius. The initial Harris sheet profile is in $z$, while $x$ and $y$ are invariant directions in the initial state. The initial magnetic field is in the $x$ direction, and $y$ is the direction of the current flow. In two of our simulation runs (cases 2, 3), we superimpose a magnetic flux perturbation to enforce an $x$-line in the center $(x, z)$ of the box (invariant in $y$ ):

case $2: \Psi_{\text {pert }}=-\Psi_{0} \cos (2 \pi x / L) \cos (\pi z / L)$

where $L=12 L_{z}$ corresponds to the length of simulation box. $\Psi_{\text {pert }}$ corresponds to the perturbed flux and $\Psi_{0}$ is the amplitude of perturbation. Here we choose a flux perturbation of $20 \%\left(\Psi_{0}=0.2 B_{0} 2 L_{z}\right)$. Let us note that the fluxfunction $\Psi$ corresponds to the $y$ component of the magnetic vector potential $A_{y}$.

In case 3 , we enforce an $\mathrm{X}$ point in the center of the simulation box by a magnetic flux perturbation structured in the $y$ direction:

case $3: \Psi_{\text {pert }}=-\Psi_{0} \cos (2 \pi x / L) \cos (\pi z / L) \cos (2 \pi y / L)$

In case 3 , we use a flux perturbation of $33 \%$. There are neutral lines at $x=0$ and $x=+-L / 2$, which change character from X-line to O-line at $|y|=L / 4$, such that in the center, at $x=0$, there is an X-line for $|y|<L / 4$ and an O-line for $|y|>L / 4$. The reverse happens at $|x|=L / 2$. This choice enforces a 3D structure. We also initialized a pure Harris sheet without any perturbation to investigate spontaneous 3D reconnection (case 4 ).

Note, that due to the necessity of resolving the velocity space (Vlasov) or particles (PIC) in kinetic simulations, the amount of memory is incomparably larger than in MHD for comparable spatial size. The tradeoff in the more realistic kinetic approach is a restriction on the size of the simulation box.

\section{Brief description of the Vlasov code}

The case 1 simulations were carried out with a newly developed Vlasov-code. We describe its main features and parameters in this section. The basic kinetic equations describing the evolution of plasmas is the Vlasov equation

$\frac{\partial f_{j}}{\partial t}+\boldsymbol{v} \cdot \nabla f_{j}+\boldsymbol{F} \cdot \nabla_{u} f_{j}=0$

where $f_{j}=f_{j}\left(x, z, v_{x}, v_{y}, v_{z}\right)$ is the distribution function and the index $j$ stands for both ions $f_{i}$ and electrons $f_{e} . \boldsymbol{F}=$ $\left(q_{j} / M_{j}\right)(\boldsymbol{E}+\boldsymbol{v} \times \boldsymbol{B})$ is the Lorentz force.

PIC codes like GISMO solve for the motion of macroparticles, while a Vlasov code directly solves Eq. (4) for electrons and ions, respectively.

While the full Vlasov equation is 6 dimensional, we assume spatial invariance in the $y$-direction. This leads to 5 dimensional equations ( 3 dimensions in velocity space and 2 dimensions in configuration space).

The Vlasov equations have to be solved selfconsistently with the Maxwell equations

$\nabla \cdot \boldsymbol{B}=0, \nabla \cdot \boldsymbol{E}=\sigma, \nabla \times \boldsymbol{B}=\boldsymbol{j}, \quad \nabla \times \boldsymbol{E}=-\frac{\partial \boldsymbol{B}}{\partial t}$,

where $\boldsymbol{B}$ is the magnetic field, $\boldsymbol{E}$ the electric field, $\sigma$ the charge density and $\boldsymbol{j}$ the current density.

With help of the magnetic vector potential $\boldsymbol{A}$ and the electric potential $\phi,(\boldsymbol{B}=\boldsymbol{\nabla} \times \boldsymbol{A}$ and $\boldsymbol{E}=-\nabla \phi-\partial \boldsymbol{A} / \partial t)$, we derive the Poisson equations from Maxwell equations:

$-\Delta \boldsymbol{A}=\boldsymbol{j}, \quad-\Delta \phi=\sigma, \quad \nabla \cdot \boldsymbol{A}=0$

The charge density $\sigma$ and the current density $\boldsymbol{j}$ are calculated by moments of the distribution functions $f_{i}$ and $f_{e}$ :

$\sigma=q_{i} \int f_{i} d^{3} v+q_{e} \int f_{e} d^{3} v$

$\boldsymbol{j}=q_{i} \int \boldsymbol{v}_{i} f_{i} d^{3} v+q_{e} \int \boldsymbol{v}_{e} f_{e} d^{3} v$

We solve the Poisson equations with the help of an implicit Gauss-Seidel matrix solver (e.g. Faires and Burden, 1993). We use Dirichlet boundary conditions in the $z$-direction and periodic boundary conditions in the $x$-direction. The time integration of the Vlasov equation is carried out by a Leap frog scheme. The boundary conditions are the same as in Poisson equations. The integration time step $\Delta t$ has to be sufficiently small to fulfill the CFL-condition for explicit time integrations, but not too small to avoid numerical diffusion (e.g. Potter, 1973).

We normalize the magnetic field by the maximum lobe field $B_{0}$, the vector potential $A$ by $B_{0} L_{z}$, the helicity density $h$ by $B_{0}^{2} L_{z}$, the mass by the proton mass $M_{i}$, the time $t$ by ion gyro frequency $\Omega_{c i}=B_{0} / M_{i}$, the velocity $v_{j}$ by $L_{z} \Omega_{c i}$, the thermal energy $T_{j}$ by thermal velocity $M_{i} v_{T j}^{2}$, the 
electric field $\boldsymbol{E}$ by $B_{0} L_{z} \Omega_{c i}$, the current density $\boldsymbol{j}$ by $B_{0} / L_{z}$, the charge density by $E / L_{z}$ and the electric potential $\Phi$ by $E L_{z}$. The distribution functions $f_{j}$ are normalized by the condition

$$
\int \exp \left(-\frac{M_{j}}{2 T_{j}}\left(v_{x}^{2}+\left[v_{y}-u_{d y j}\right]^{2}+v_{z}^{2}\right)\right) d^{3} v=1
$$

The Vlasov-simulation results, presented here in this paper, use a reduced mass ration of $M_{i} / M_{e}=16$ and a temperature ratio of $T_{i} / T_{e}=1$. We use a spatial grid of 40 grid points in $x$ and 20 grid points in $z$. The box length is $-8 L_{z}<x<8 L_{z}$ and $-4 L_{z}<z<4 L_{z}$. The temperature is $T_{i}=T_{e}=0.3$ and the Debye Length for both ions and electrons is $0.54 L_{z}$ and thus, the Debye length is resolved by 1.4 grid points. We resolve the phase space by $10 \times 10 \times 10$ grid points and a box length in phase space of 6 thermal velocities $\left(-3 v_{T j}<v_{j}<3 v_{T j}\right)$. Notice that the thermal velocities of ions and electrons are different by a factor of 4 for our parameter set $\left(v_{T e}=\sqrt{M_{i} / M_{e}} \sqrt{T_{e} / T_{i}} v_{T i}\right)$ and thus, we use different grids in phase space for the distribution function of ions and electrons. This is justified since the Vlasov-equations for ions and electrons are only coupled by the electromagnetic field. We resolve the ion gyro time $\Omega_{c i}^{-1}$ by 130 time steps and the electron gyro time by 8 time steps.

As initial configuration we use a perturbed Harris sheet profile (2) with $\epsilon_{1}=0.05$ and $\epsilon_{2}=1$ (X-point in center of sheet). We also present results of simulations with a longer box in the $x$-direction $\left(-16 L_{z}<x<16 L_{z}\right)$ and $\epsilon_{2}=2$, which corresponds to an extension of the previous simulation box at both ends in order to include two O-points.

\section{Simulation results}

\subsection{Time evolution}

In this section we compare different aspects of the four investigated cases. We are mainly interested in the magnetic field structure after magnetic reconnection has occurred. Since we compare perturbed sheets, triggered and spontaneous reconnection runs, we diagnose the magnetic field component $\left|\boldsymbol{B}_{z}\right|=\sqrt{B_{z}^{2}}$, averaged over the whole box. In Fig. 1, we show the time evolution of $\left|\boldsymbol{B}_{z}\right|$ normalized to the maximal lobe field $B_{0}{ }^{1}$ for all cases. In the following presentation, we refer to fully developed reconnection when a level of $B_{z} / B_{0}$ is reached, close to its maximum and before the influence of the boundaries becomes essential. Due to the different initial states, the reconnection time differs:

$\begin{array}{llr}\text { case } 1 & \text { (2D Vlasov, 2D modified Harris sheet) } & 14 \Omega_{c i}^{-1} \\ \text { case } 2 & \text { (3D PIC, 2D perturbation) } & 5 \Omega_{c i}^{-1} \\ \text { case 3 } & \text { (3D PIC, 3D perturbation) } & 2 \Omega_{c i}^{-1} \\ \text { case } 4 & \text { (3D PIC, spontaneous) } & 22 \Omega_{c i}^{-1}\end{array}$

\footnotetext{
${ }^{1}$ We used $B_{0}$ corresponding to a maximal lobe field of $6 \cdot 10^{-4}$ Gauss and we choose a sheet width of $L_{z}=372000 \mathrm{~cm}$.
}

Reconnection is fastest in the 3D configurations with magnetic field perturbation (cases 2, 3). In these cases, the Harris sheet is not in an exact equilibrium and, therefore, more unstable than the exact equilibrium (case 4) or in the slightly modified Harris equilibrium (case 1). The modified Harrisequilibrium includes an $\mathrm{X}$-point as a seed for the occurrence of magnetic reconnection. In spontaneous reconnection, the configuration has to find this seed itself from the fluctuations. Consequently, reconnection is faster in case 1 than in the unperturbed case 4 , but slower than in configurations with magnetic field perturbation (cases 2,3). Notice that 2D VlasovCode simulations for an unperturbed Harris sheet (not shown here) lead to spontaneous magnetic reconnection after approximately $60 \Omega_{c i}^{-1}$. This is by a factor of about 2.5 slower than the occurrence of spontaneous magnetic reconnection in case 4. The magnetic reconnection process is faster in 3D because kinetic current instabilities (Büchner and Kuska, 1999) occur and couple with reconnection (Büchner, 1999; Wiegelmann and Büchner, 2000). The kinetic current instabilities are in the current direction $(y)$. Thus, they cannot occur in 2D simulations of the classical reconnection plane $x z$. Therefore, in $2 \mathrm{D}$, reconnection and current instabilities can only be investigated independently from each other; they couple in 3D. The current instabilities are not a result of reconnection, but rather the result of fast flows of the ions.

\subsection{Structure of reconnection}

In this paper, we mainly investigate the helicity evolution. The magnetic helicity can be determined as $H=\int \boldsymbol{A} \cdot \boldsymbol{B} d V$. The total magnetic helicity $H=\int \boldsymbol{A} \cdot \boldsymbol{B} d V$ is gauge invariant for configurations with $\boldsymbol{B} \cdot \boldsymbol{n}=0$ on the boundary. The latter condition is fulfilled for the Harris sheet equilibria with periodic boundary conditions investigated in this paper.

Since it contains important information about the structure of the magnetic field, we diagnose the helicity density $h=\boldsymbol{A} \cdot \boldsymbol{B} \cdot{ }^{2}$ This can be seen in analogue to fluid dynamics (e.g. Levy et al., 1990; Moffat and Tsinober, 1992), where the helicity density $\boldsymbol{v} \cdot \boldsymbol{\omega}$ contains important information regarding the flow, e.g. it helps to localize the vortex core and its axis. It can also be shown that the helicity density in axisymmetric fluid flows changes its sign across a separation line. Similar effects occur in the magnetic helicity density which helps us to identify the core of a perpendicular magnetic field $B_{y}$ and, in the 2D, case the magnetic helicity density changes its sign across a separation line which separates regions of different magnetic topology after reconnection occurred. The magnetic field is a vector and the magnetic helicity density is a scalar. Contourplots of this scalar value help to visualize and analyse important characteristics of the magnetic field. It is far more complicated to directly analyse the 3D vector field $\boldsymbol{B}$. Note the similarity of the role of flow vortex helicity density for fluid flow vorticity in hydrodynamics, and the role of magnetic helicity density for the

\footnotetext{
${ }^{2}$ Obviously this value depends on the gauge of $\boldsymbol{A}$. The structure of the helicity density does not depend on the gauge, however.
} 

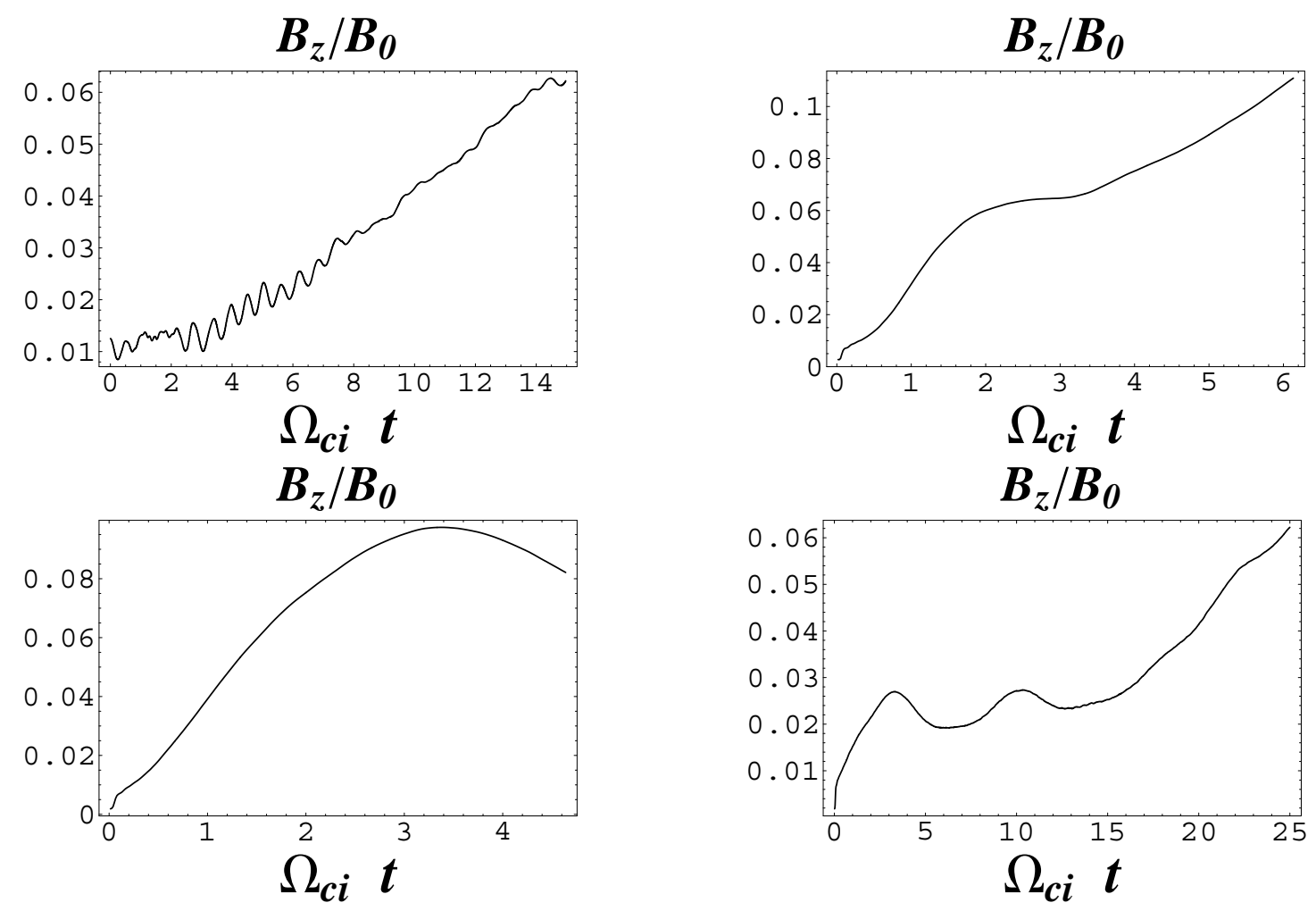

Fig. 1. $B_{z} / B_{0}$ for $2 \mathrm{D}$ (upper left, case 1), 3D with $2 \mathrm{D}$ perturbation (upper right, case 2), 3D with 3D perturbation (lower left, case 3) and spontaneous $3 \mathrm{D}$ (lower right, case 4 ), as a function of time.
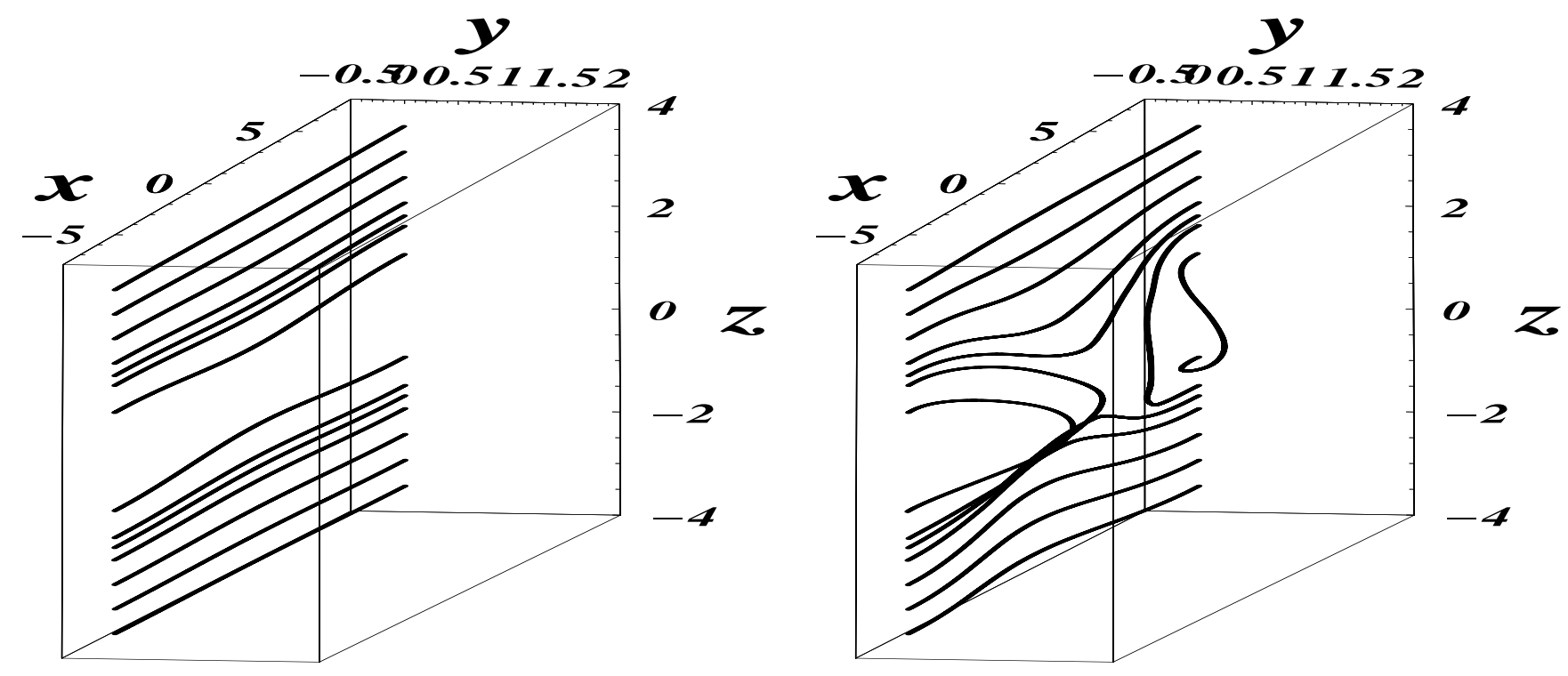

Fig. 2. Magnetic field lines for $2 \mathrm{D}$ kinetic reconnection (Vlasov-code-simulation). Initial state on the lefthand side and after magnetic reconnection had occurred, on the righthand side. As a consequence of the quadrupolar structure of $B_{y}$, the field lines become distorted perpendicular to the reconnection plane. We over rate the $B_{y}$ component by a factor of 5 , for illustration. 
magnetic field connectivity in plasmas.

\subsubsection{Case 1 (2D Vlasov)}

In pure 2D Harris sheets, which are invariant in the current (y) direction, the magnetic field obtains a component perpendicular to the reconnection plane, but no helicity change can be seen. Figure 2 shows a configuration with an X-point and Fig. 4, a configuration with two additional O-points (longer box, accommodating two tearing mode wavelength). As one can see in Figs. 2 and 4, the initial plane sheet (left-hand side of the figures) becomes bend in the current direction $(y)$ after magnetic reconnection has occurred, as predicted by Terasawa (1983). This bending corresponds to the formation of a quadrupolar distribution of the magnetic field component $B_{y}$ which arises from the Hall effect of different mobilities, resulting in a quadrupolar structure of the helicity density around the X-line (Fig. 5 upper left and right panel).

The occurrence of the quadrupolar structure can be explained as follows. Due to the difference mass between electrons and ions, the mobility of electrons and ions is different. Consequently, the particle flux of ions and electrons out of the reconnection zone (X-point) is different (see Fig. 3, lower panels). The ions are streaming mainly parallel to the $x$-axis, but the electrons are along the wings of the magnetic field. The different particle flows cause four ring currents around the central X-point (see Fig. 3, upper right panel). Each of these ring currents in the $x z$-plane naturally causes a magnetic field $B_{y}$ perpendicular to the reconnection plane (see Fig. 3, upper left panel). Due to the orientation of the four ring currents, we obtain the observed quadrupolar structure in $B_{y}$. In 2D, we have approximately $\boldsymbol{A} \cdot \boldsymbol{B} \approx A_{y} B_{y}$ and, consequently, the helicity density also contains a quadrupolar structure.

Notice that, since the effect of bending is small, we over rate the magnetic field component $B_{y}$ by a factor of 5 , in Figs. 2 and 4 , to illustrate the effect more clearly. The further plots correspond to the shorter simulation box (only one $\mathrm{X}$-line, our case 1, see Fig. 2). Figure 5, shows in the upper left panel, a contourplot of the magnetic field component in the current direction $B_{y}$, which was developed as a consequence of magnetic reconnection. As one can see, this magnetic field component contains a pure quadrupolar structure. The quadrupolar structure of $B_{y}$ corresponds to a quadrupolar structure in the helicity density as presented in the upper right panel of Fig. 5. Meanwhile, the total helicity is approximately conserved; this can be seen in the upper left panel of Fig. 10 where the thick line shows the overall helicity. The dotted line in the same panel corresponds to the evolution of the integrated helicity density of the upper right and lower left corners of the reconnection plane, and the dashed line, to the evolution of the integrated helicity density in the opposite corners. The amplitudes are both increasing during the reconnection process, but they are anti-symmetric, so that the total magnetic helicity remains constant. As pointed out by Hornig (1999a), cases of magnetic reconnection with $\boldsymbol{E} \cdot \boldsymbol{B}=0$ conserve the magnetic helicity exactly. When $\boldsymbol{E} \cdot \boldsymbol{B}=0$ no parallel electric fields $E_{\|}=\boldsymbol{E} \cdot \boldsymbol{B} /|\boldsymbol{B}|$ evolve during the reconnection process. Indeed, one can see, in the lower left panel of Fig. 5, the parallel electric field is very weak by a factor of about 35 smaller than the reconnection electric field $E_{y}$ (lower right panel). Consequently, the magnetic helicity is practically conserved. Let us note that $E_{y}$ reaches its maximum at the X-point, as expected for $2 \mathrm{D}$ reconnection. The very weak parallel electric field has a quadrupolar symmetry and reaches its maximum in the wings of the reconnection zone. In $2 \mathrm{D}$ reconnection, the magnetic field has a singularity at the X-points (here, $B_{y}$ vanishes as well). This corresponds to classical magnetic field line merging (Vasyliūnas, 1975), which is a special case of general magnetic reconnection, known as $\boldsymbol{B}=0$ reconnection (Schindler et al., 1988).

\subsubsection{Remarks on cases $2-4$}

In 3D (cases 2-4), kinetic current instabilities occur in addition to the pure tearing instability (Büchner, 1999). The coupling of these current instabilities and tearing dominates in spontaneous reconnection (case 4; Wiegelmann and Büchner, 2000). To obtain some insights regarding the structure of 3D reconnection, we try to find a rather smooth transition from pure 2D reconnection (case 1) to full 3D reconnection (case 4). Such an intermediate case would consist of a process where current instabilities are available, but not dominating. While the growth rate of the drift current instability (Büchner and Kuska, 1999) is controlled by the physical system, although it is not affected by the initial magnetic field perturbation, we can externally encourage the reconnection process with the help of external magnetic field perturbations. This ensures that the wave amplitude of drift current instabilities is smaller for driven 3D reconnection than for spontaneous $3 \mathrm{D}$ reconnection. Reconnection goes faster in case 3 than in case 2 and, consequently, the role of kinetic current instabilities is more important in case 2 than in case 3 .

To compare cases 2-4 (3D PIC) with case 1 (2D simulations), we present the fields in the $x z$-reconnection plane at the center of the current direction $(y=0)$, and present the same quantities ( $B_{y}$, helicity density, $\left.E_{\|}, E_{y}\right)$, as in case 1 .

\subsubsection{Case 2 (3D PIC, 2D perturbation, Fig. 6)}

Both $B_{y}$ and the helicity density exhibit a quadrupolar structure (upper panels). It is, however, somewhat fuzzy compared with the pure 2D case, as a result of the occurrence of a kinetic current instability. As one can see, the magnetic field does not vanish completely at the X-point. Thus, we do not observe 2D magnetic merging, but a more general reconnection, which is defined by changes of magnetic connectivity (Axford, 1984). While in MHD, the localized breakdown of the frozen-in field condition would need an ad hoc resistivity; the system finds its resistivity itself within the framework of the kinetic approach used here in this work. The small finite magnetic field component $B_{y}$, in the current direction, leads to $\boldsymbol{B} \neq 0$ everywhere in the reconnection re- 

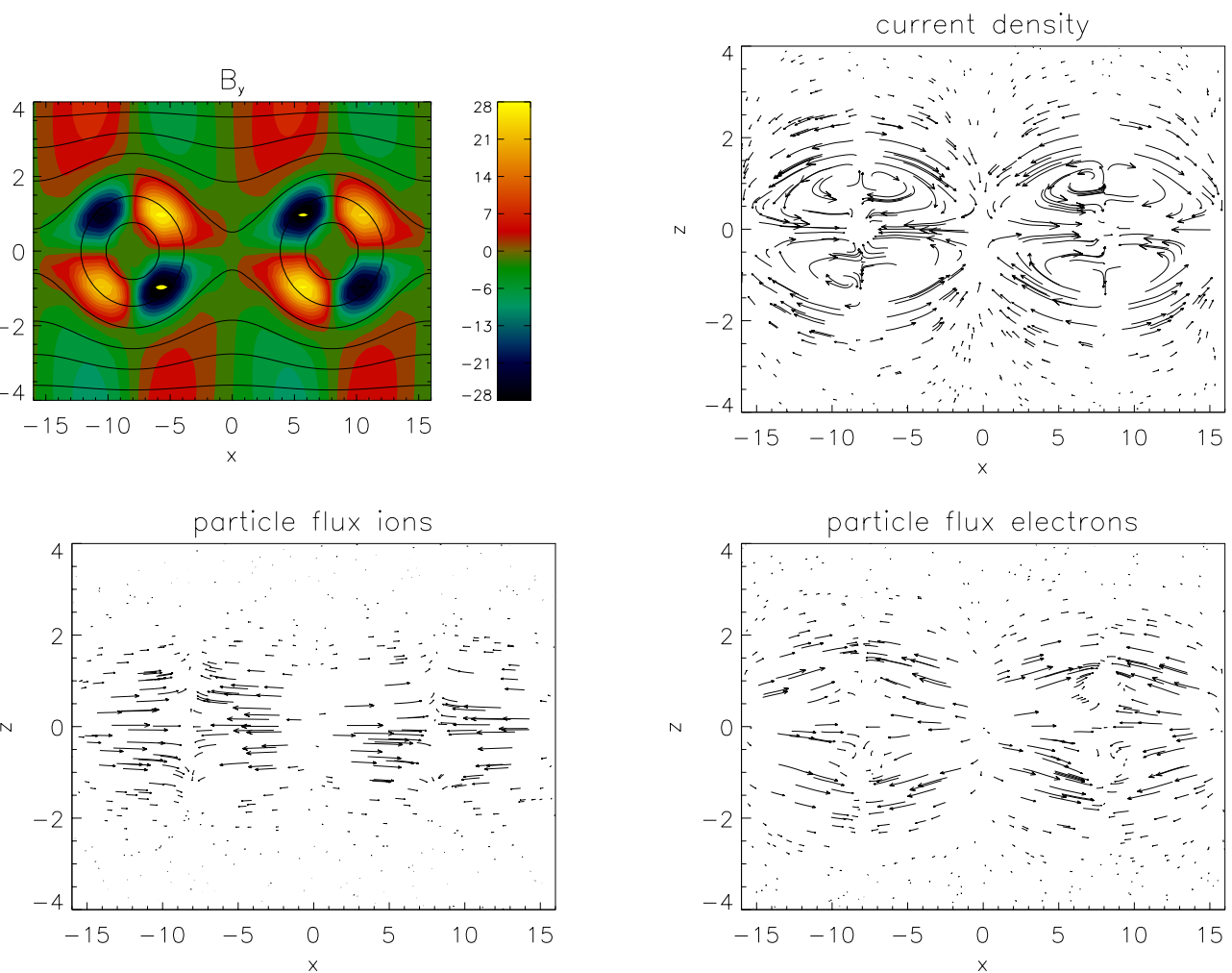

Fig. 3. The upper left panel shows a contourplot of magnetic field component $B_{y}$ (in $10^{-6} \mathrm{G}$ ) correspondent to the magnetic field configuration in Fig. 4, right panel. The contour lines correspond to a projection of magnetic field lines in the plane. The three other panels correspond to the flow of the current density, the particle flux of ions, and particle flux of electrons, respectively.

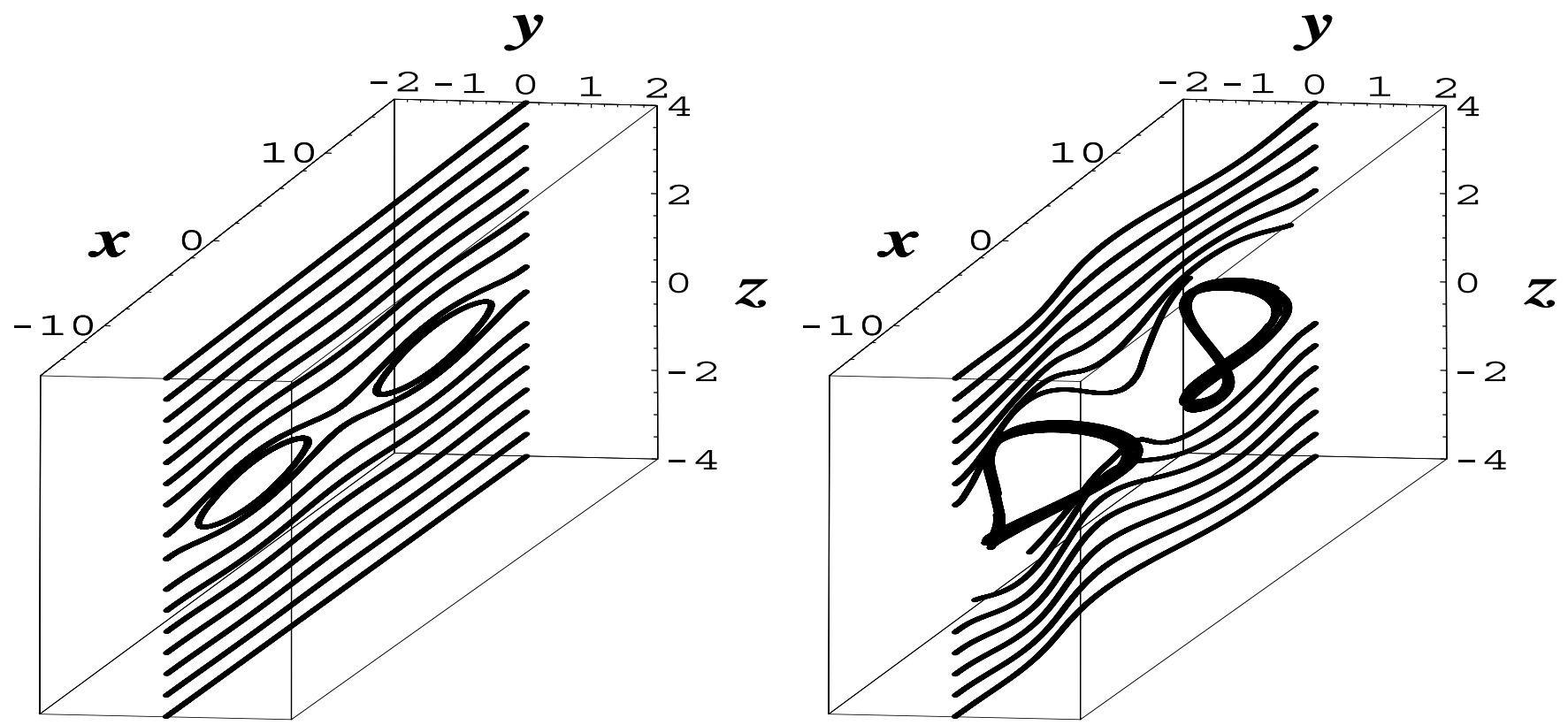

Fig. 4. Magnetic field lines for 2D reconnection (Vlasov-simulation). Initial state on the left-hand side and after magnetic reconnection has occurred on the right-hand side. Here, we extended the simulation box by a factor of two in the $x$-direction to include two tearing mode wavelength (i.e. two O-points). As a result of the quadrupolar structure of magnetic helicity density, the field lines become distorted in the $y$ direction. The effect is small and we over rate the $B_{y}$ component by a factor of 5, for illustration. 

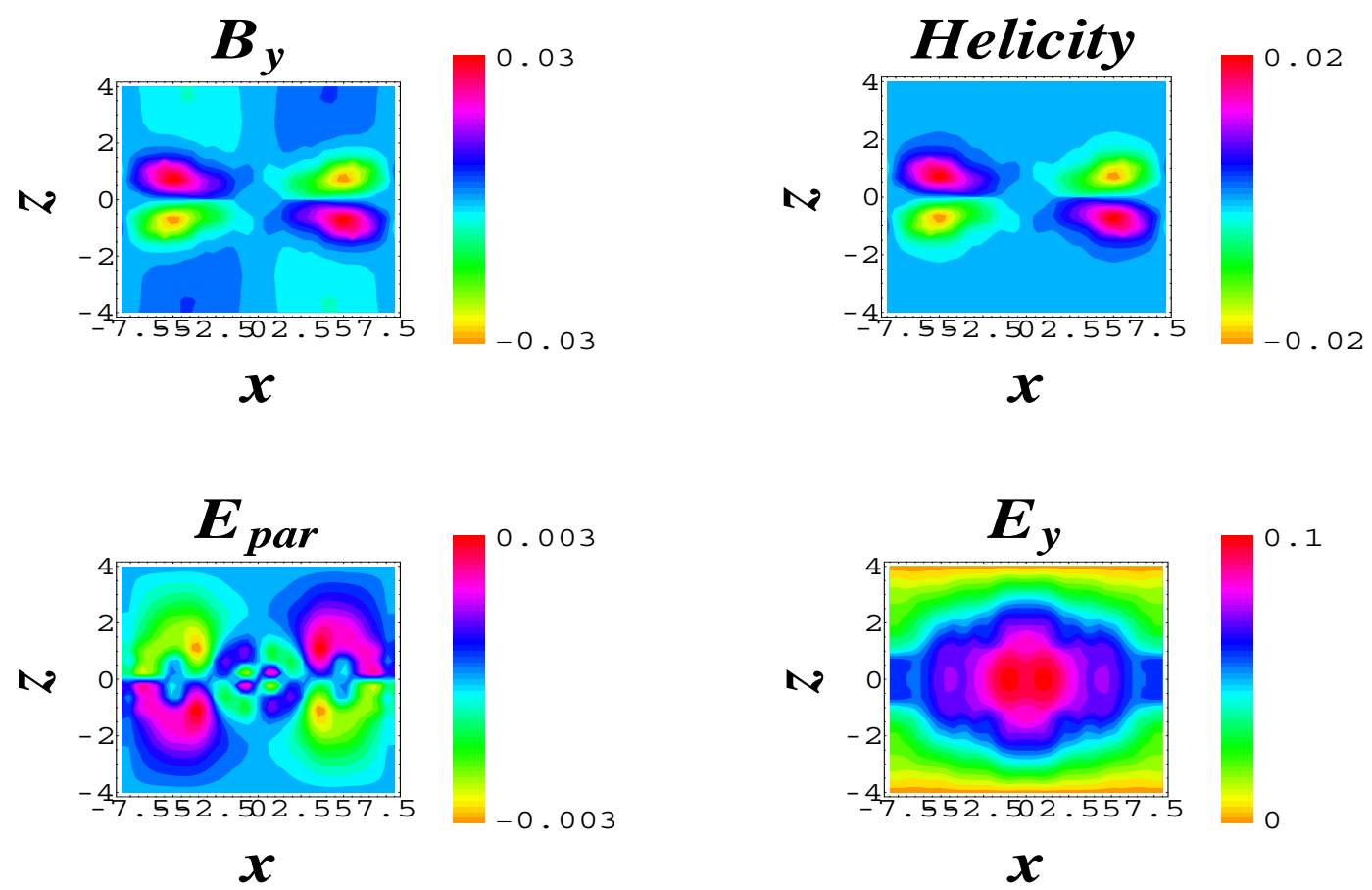

Fig. 5. Case 1: Perpendicular magnetic field (in $10^{-3} \mathrm{G}$ ), helicity density (in $\mathrm{G}^{2} \mathrm{~cm}$ ), parallel electric field (in $10^{-3} \mathrm{CGS}_{[\mathrm{E}]}$ ) and perpendicular electric field (in $10^{-3} \mathrm{CGS}_{[\mathrm{E}]}$ ) after magnetic reconnection occurred in the framework of $2 \mathrm{D}$ Vlasov-simulations.
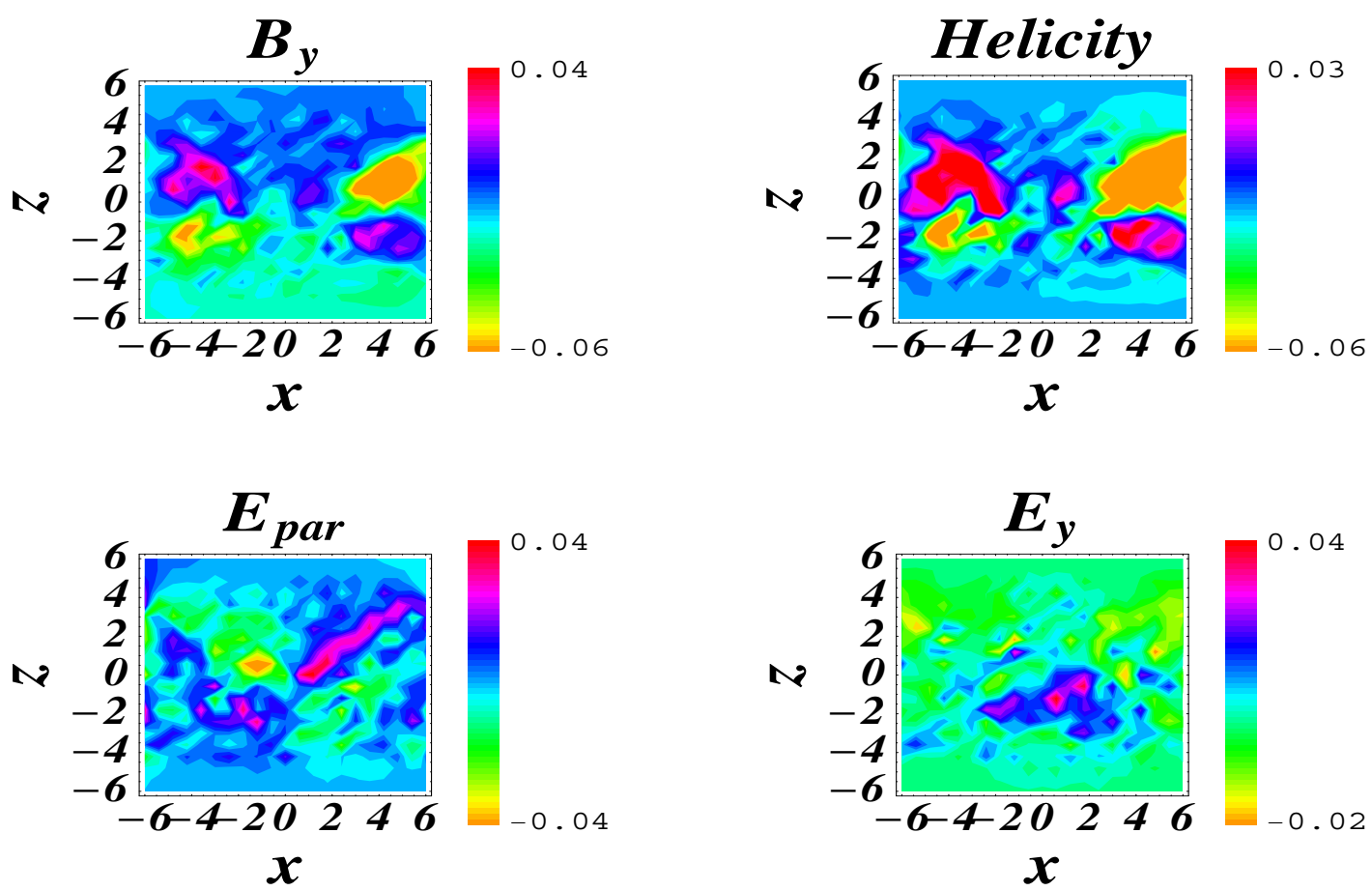

Fig. 6. Case 2: Perpendicular magnetic field (in $10^{-3} \mathrm{G}$ ), helicity density (in $\mathrm{G}^{2} \mathrm{~cm}$ ), parallel electric field (in $10^{-3} \mathrm{CGS}[\mathrm{E}]$ ) and perpendicular electric field (in $10^{-3} \mathrm{CGS}_{[\mathrm{E}]}$ ) after magnetic reconnection has occurred in the framework of 3D PIC-simulations with a 2D magnetic field perturbation. 

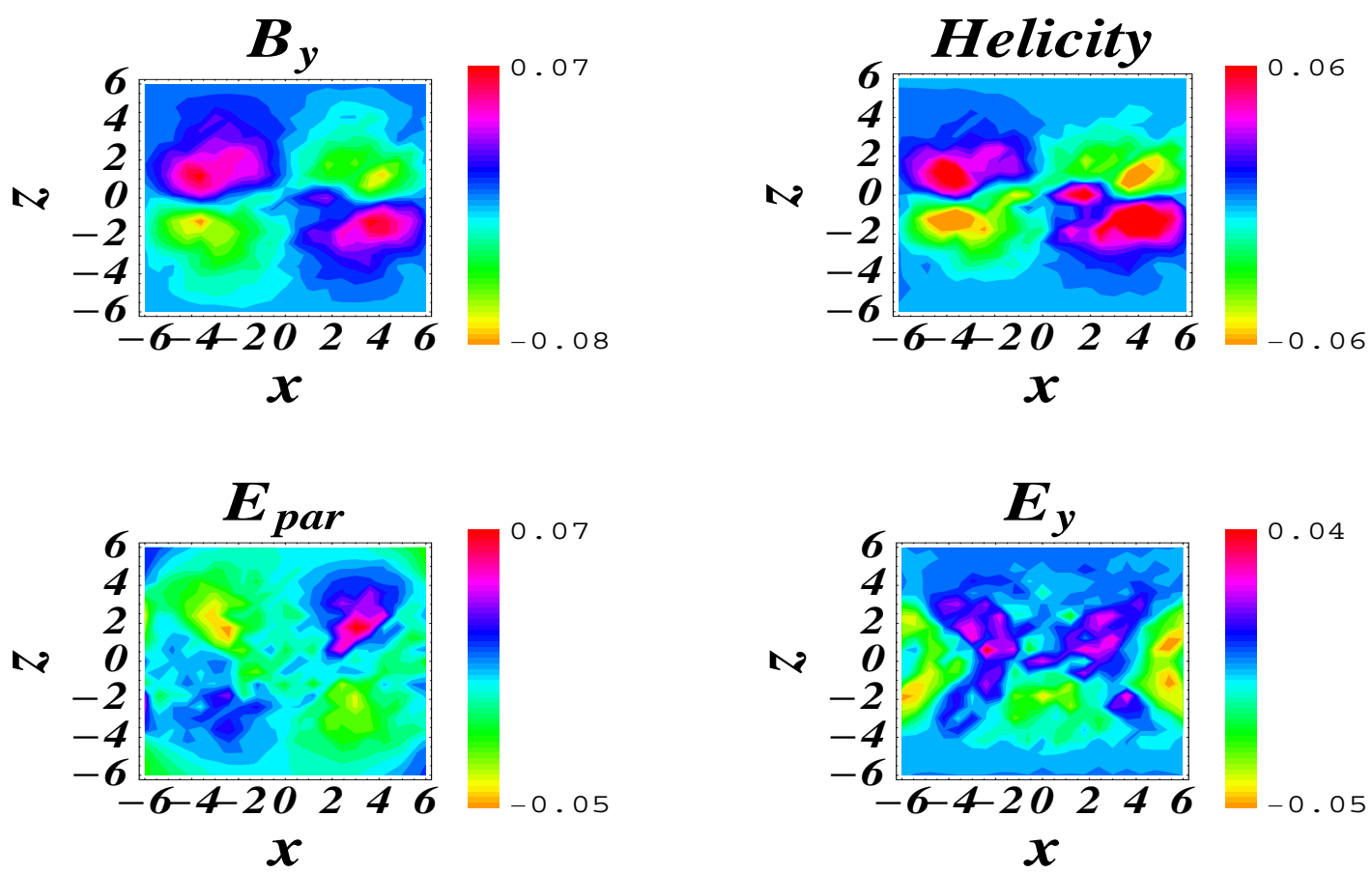

Fig. 7. Case 3: Perpendicular magnetic field (in $10^{-3} \mathrm{G}$ ), helicity density (in $\mathrm{G}^{2} \mathrm{~cm}$ ), parallel electric field (in $10^{-3} \mathrm{G}$ ) and perpendicular electric field (in $10^{-3} \mathrm{CGS}_{[\mathrm{E}]}$ ) after magnetic reconnection has occurred in the framework of 3D PIC-simulations with a 3D magnetic field perturbation.
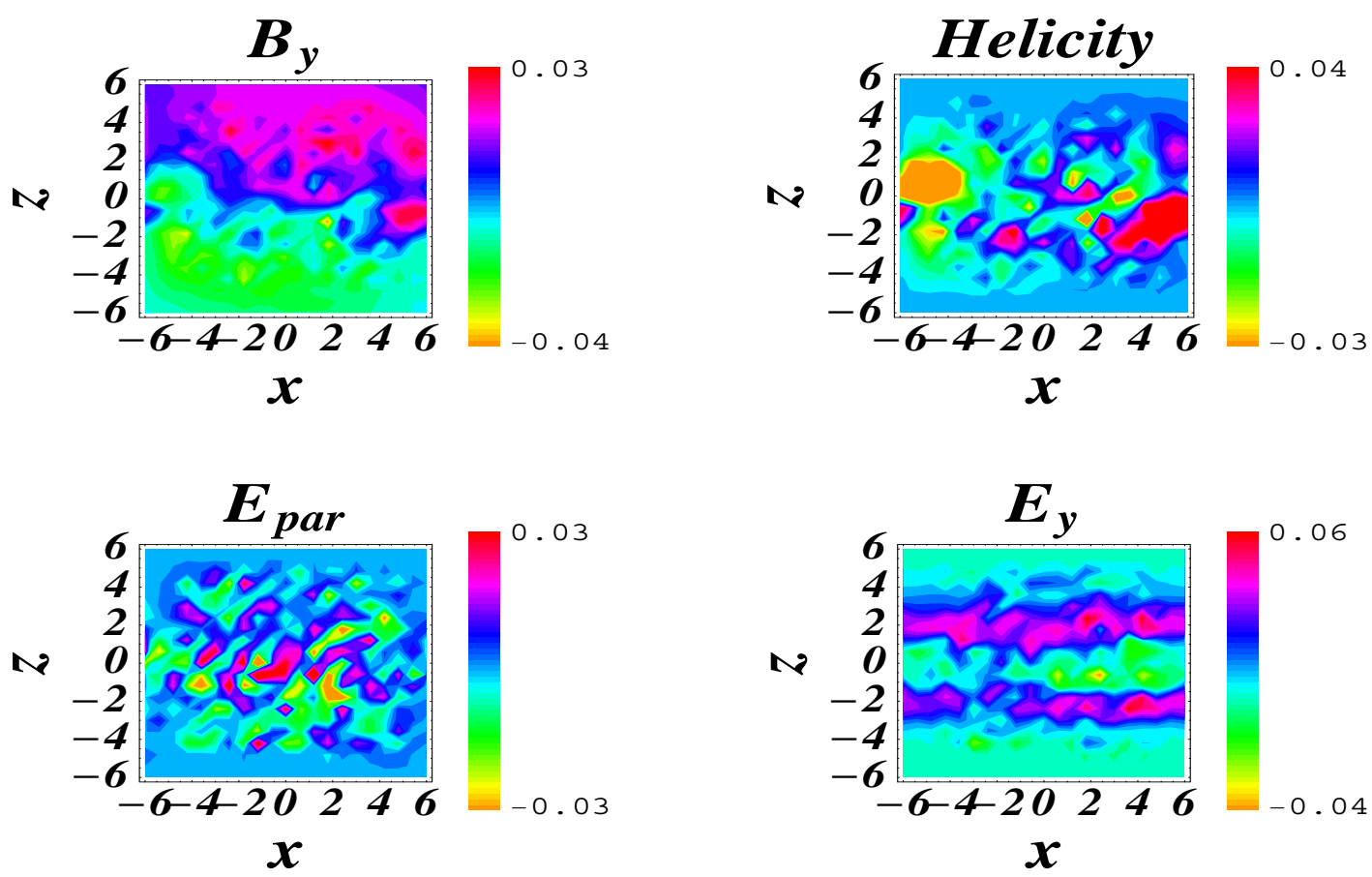

Fig. 8. Case 4: Perpendicular magnetic field (in $10^{-3} \mathrm{G}$ ), helicity density (in $\mathrm{G}^{2} \mathrm{~cm}$ ), parallel electric field (in $10^{-3} \mathrm{CGS}[\mathrm{E}]$ ) and perpendicular electric field (in $10^{-3} \mathrm{CGS}_{[\mathrm{E}]}$ ) after magnetic reconnection has occurred in the framework of spontaneous 3D PIC-simulations without any perturbations. 
gion, in case for general magnetic reconnection (Schindler et al., 1988). One finds a finite $E_{\|}$of the same order of magnitude as $E_{y}$. The very regular structure of the reconnection electric field, as seen in the 2D-case, disappears in the 3D case. The upper right panel of Fig. 10 shows the time evolution of the helicity density, integrated over the diagonal opposite wings of the reconnection plane. The helicity density in the wings changes as in the pure $2 \mathrm{D}$ case. The total helicity is not exactly conserved here, but exact conservation of helicity is only expected for $\boldsymbol{B}=0$ reconnection, while for finite $\boldsymbol{B}$ reconnection, the helicity may slowly dissipated. We find, however, that the total helicity is still approximately conserved. Finite $\boldsymbol{B}$ reconnection allows $\boldsymbol{E} \cdot \boldsymbol{B} \neq 0$ (finite $\left.E_{\|}\right)$.

\subsubsection{Case 3 (3D PIC, 3D perturbation, Fig. 7)}

The structure of $B_{y}$ and the helicity density, shown in the upper panel of Fig. 7, is less fuzzy here than in case 2. Instead, it resembles the pure quadrupolar structure in case 1 (2D Vlasov). The reason is that due to the fast reconnection, the current instability does not succeed in growing to an essential amplitude. A main difference is, however, that $E_{\|}$, which was negligible in case 1, dominates here (lower panels). The parallel electric fields even exceed $E_{y}$. While the structure of $E_{\|}$is quadrupolar similar to the 2D case, the amplitude of the parallel electric field is by a factor of 20 higher. The total helicity is approximately conserved during the time evolution, as presented by the thick solid line in the lower left panel of Fig. 10.

\subsubsection{Case 4 (3D spontaneous reconnection, Fig. 8)}

As one sees in the upper panels of Fig. 8, in the case of spontaneous 3D-reconnection, neither $B_{y}$ nor the helicity density contains the quadrupolar structure, as observed in cases 1-3. Instead, there seems to be a dipole structure for both $B_{y}$ and the helicity. In contrast to the other three cases of triggered reconnection, however, the structure of $B_{y}$ and helicity density are not similar. The helicity density in the wings, which would correspond to a quadrupolear structure, is oscillating (lower right panel of Fig. 10). This oscillation corresponds to the current driven unstable wave moving through the structure. The coupling of kinetic current instabilities and reconnection dominates here. The helicity is only approximately conserved, as in cases 2 and 3; this corresponds to the development of a finite $E_{\|}$component, i.e. a finite magnetic field component $B_{y}$ (lower left panel of Fig. 8). The amplitude of the parallel electric field is approximately half of $E_{y}$ presented in the lower right panel of Fig. 8. The intrinsic 3D structure of magnetic reconnection (Büchner and Kuska, 1996; Pritchett et al., 1996; Büchner, 1999; Zhu and Winglee, 1996), which occurs in case 4 , can certainly not contain very special constraints, such as a vanishing magnetic field in the reconnection region, and a corresponding vanishing parallel electric field (or $\boldsymbol{E} \cdot \boldsymbol{B}=0$ ), as in case 1. While these constraints are necessary to ensure an exact conservation of magnetic helicity, the helicity can slowly dissipate as a result of $3 \mathrm{D}$ magnetic reconnection.

\subsection{Diagnostics regarding quadrupolar and dipolar struc- ture}

Let us now concentrate on the question of whether we observe a quadrupolar or dipolar structure in the helicity density in the four different cases. In order to do so, we show the time evolution of the different contributions to the helicity in Fig. 11. The solid lines depict the quadrupolar component, the thick dotted line depicts the dipolar component with asymmetry in $x$, and the dashed line depicts the dipolar component with asymmetry in $z$. For the 2D case (upper left panel), we observe a pure quadrupolar structure and no dipolar component of the helicity structure. A quadrupolar part of the helicity is also always available in the 3D cases, but the structure also contains a dipolar component in 3D. In all investigated cases, the $x$ dipolar component is stronger than the $z$ dipolar one; its amplitude is only somewhat lower than the quadrupole part of the helicity density structure for case 2 (3D with 2D-perturbation, upper right panel of Fig. 11). For case 3 (3D with 3D-perturbation, lower left panel of Fig. 11) this dipolar part is available as well, but its amplitude is far lower than the amplitude of the quadrupole. We note that the absolute amplitude of the quadrupolar component is by a factor of 4 larger than in the other cases, and the absolute dipolar amplitude is of the same order of magnitude for cases 2 and 3. For case 4 (spontaneous 3D without any perturbation, lower right panel of Fig. 11), we can still identify a quadrupolar part of the helicity structure; its absolute amplitude in the reconnected state is of the same order of magnitude as in cases 1 and 2. The amplitude of the main dipolar part, however, is dominating by a factor of about 3 over the quadrupole amplitude. The dipolar component's amplitude oscillates in correspondence with the drift current instability moving through the structure. The dipolar part in the helicity structure, which is present in all 3D simulations (and thus, in all cases where drift current instabilities occur) explains the differences in the helicity density structure between pure 2D and $3 \mathrm{D}$ reconnection. Due to the overwhelming drift current instabilities and thus, the overwhelming dipole structure in the helicity structure, naked eye observation, e.g. Fig. 8, do not show the quadrupole component, although it is always present.

\subsection{D helicity density structure}

While we have shown the helicity density structure in the center plane $y=0$ in the upper right panels of Figs. 5-8 for the four investigate cases, we present the 3D structure of the helicity density in Fig. 9. Here, we show 3D equicontour surfaces after reconnection has occurred (same times as in the $2 \mathrm{D}$ cuts). The red surfaces correspond to a positive helicity and the green surfaces correspond to a negative helicity. (The surfaces correspond to a helicity density of $30 \%$ of the maximum amplitude.) 

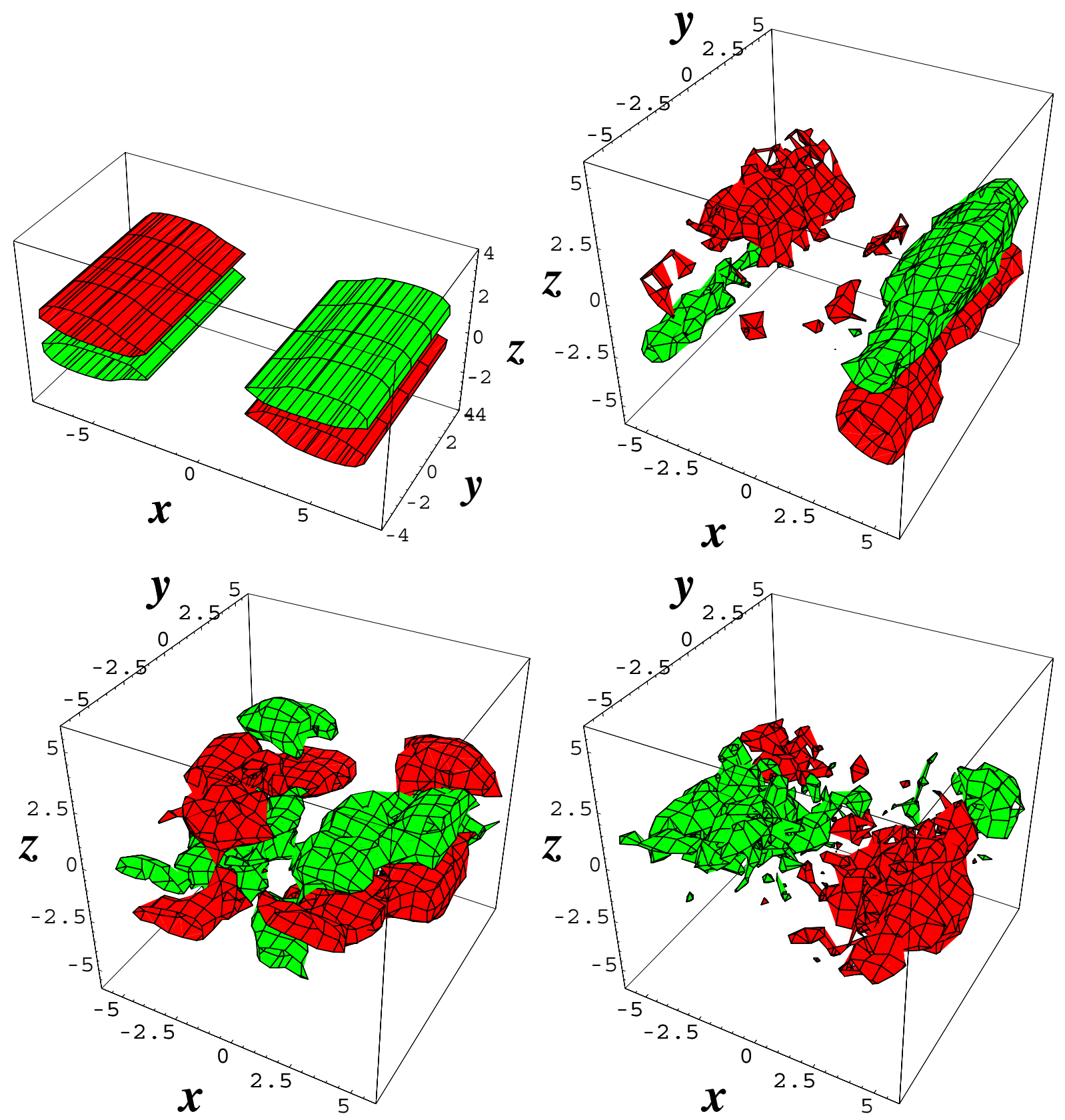

Fig. 9. Equicontourplots of magnetic helicity density (positive red, negative green, 0.3 of maximum) for 2D (upper left), 3D with 2D perturbation (upper right), 3D with 3D perturbation (lower left) and spontaneous 3D (lower right) after reconnection has occurred.

- Case 1 (2D Vlasov, Fig. 9 upper left panel): The panel shows the pure quadrupolar structure, invariant in $y$, due the $2 \mathrm{D}$ approach $(\partial / \partial y=0)$.

- Case 2 (3D PIC with 2D perturbation, Fig. 9 upper right panel): The quadrupolar structure is still present here, but the helicity densities are becoming structured in the current direction $(y)$, as a result of a current instability. The structure is no longer purely quadrupolar, as mentioned before, while the naked eye observations would still speak in favour of a fuzzy quadrupole.
- Case 3 (3D PIC with 3D perturbation, Fig. 9 lower left panel): The helicity structure becomes more complex, due to the 3D magnetic field perturbation. The pure quadrupole is present only in the plane $y=0$ (similar to cases 1 and 2). The helicity density changes its sign along the y-axis. This is certainly not an intrinsic effect of the reconnection process itself, but obviously occurs due to the construction of the perturbing magnetic field, which enforces the 3D structure.

- Case 4 (3D PIC spontaneous, Fig. 9 lower right panel): Here the helicity density shows a somewhat fuzzy dipole, 

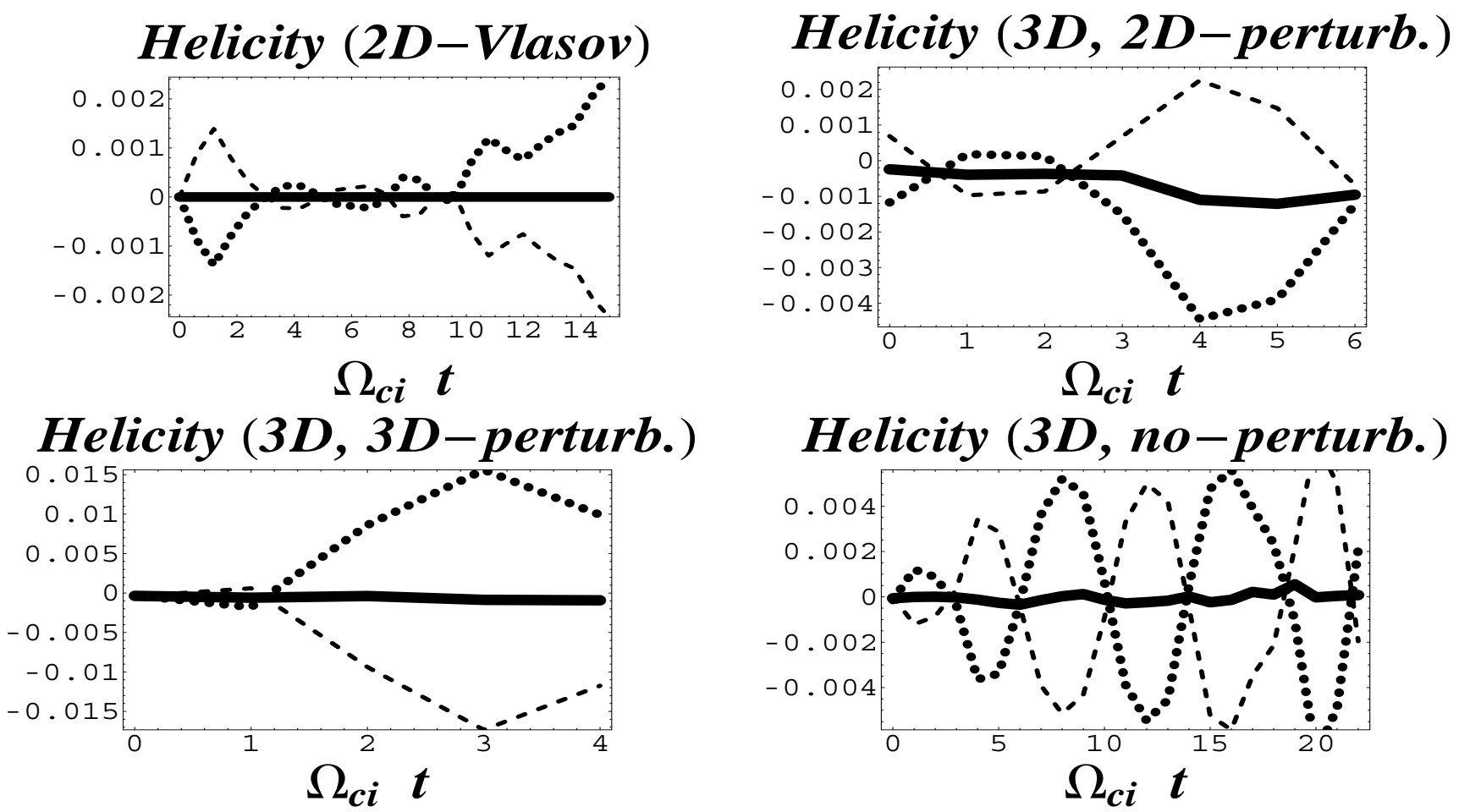

Fig. 10. Time evolution of the magnetic helicity (in $\mathrm{G}^{2} \mathrm{~cm}$ ) for the different cases (solid lines). The dashed lines correspond to the integrated helicity density in the upper left and lower right part, the dotted lines to the integrated helicity density in the upper right and lower left part and the thick solid line corresponds to the total helicity, which is approximately conserved.
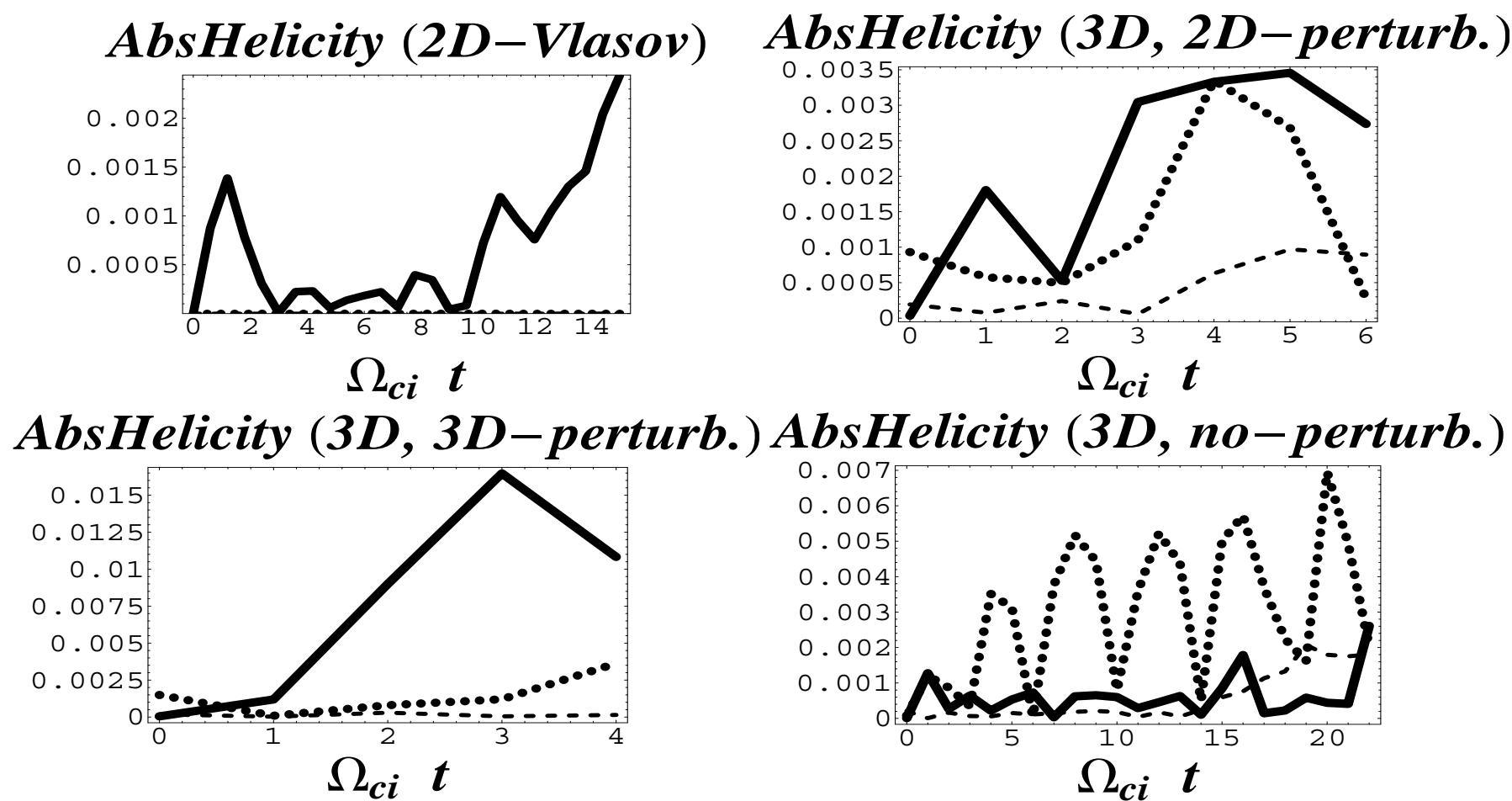

Fig. 11. Time evolution of the absolute amplitudes of helicity structure (in $\mathrm{G}^{2} \mathrm{~cm}$ ) for the different cases. The solid lines correspond to the quadrupolar part of the helicity density structure, the dotted lines correspond to the dipole part with left-right asymmetry and the (thin) dashed line corresponds to the dipole part with up-down asymmetry. Notice that in pure 2D magnetic reconnection, as presented in the upper left panel, the helicity has a pure quadrupolar structure without any dipole parts. 
asymmetric in $x$, because the dipolar part of the helicity density is clearly dominating, as a result of the evolution of the current instability.

\section{Conclusions and outlook}

In this paper, we investigated the helicity evolution in $2 \mathrm{D}$ and 3D kinetic reconnection, with the help of numerical simulations, using a Vlasov code in 2D and PIC simulations in 3D. We start with a Harris type current sheet, with a sheet width comparable to the ion gyroradius. Our results confirm the prediction of Terasawa (1983), that in 2D, the magnetic structure exhibits a pure quadrupolar structure in the reconnection region, and the magnetic field becomes distorted perpendicular to the reconnection plane. The overall helicity is conserved. In accordance with the general magnetic reconnection investigations (Schindler et al., 1988), the parallel electric field and the magnetic field vanishes in the 2D reconnection region $(\boldsymbol{B}=0$ reconnection, $\boldsymbol{E} \cdot \boldsymbol{B}=0$ reconnection ). While the helicity conservation was predicted for this special case of magnetic reconnection within the framework of MHD investigations, it certainly could not explain the local helicity density structure around the X-point. The situation becomes more difficult in 3D. Here, additional drift current instabilities occur in the current direction (Büchner and Kuska, 1999) and couple with the magnetic reconnection process (Büchner, 1999; Wiegelmann and Büchner, 2000). The consequence for the local helicity density distribution is that a dipolar magnetic structure additionally evolves in 3D, which eventually becomes dominating. 3D reconnection is characterized by essential amounts of parallel electric fields. As a result, the overall helicity is no longer conserved, as in 2D $(\boldsymbol{B}=0$ and $\boldsymbol{E} \cdot \boldsymbol{B}=0)$. While the concept of general magnetic reconnection within the framework of MHD just assumes an intrinsic 3D magnetic field in the reconnection region, our kinetic simulations of thin current sheets identified kinetic current instabilities as its possible cause. Our simulations also confirm parallel electric fields and slow time variation of the total helicity. Additionally, they give insights into the local helicity density structure in the reconnection region and identify physical reasons for different behaviour of 2D and 3D magnetic reconnection. As a next step, we will investigate the influence of an external magnetic shear component, $B_{y}$, in the initial Harris sheet configuration. The results will be presented in a subsequent paper.

Acknowledgements. We thank Jens-Peer Kuska for developing the PIC-code GISMO and Rainer Grauer, Andreas Kopp and Bernd Nikutowski for useful discussions. We thank Profs. Sami Solanki and Vytenis Vasyliūnas for supporting this work at the Max-PlanckInstitut für Aeronomie in Katlenburg-Lindau. We thank Joachim Birn and an unknown referee for their useful comments.

\section{References}

Axford, W. I., Magnetic field reconnection, in Reconnection in space and laboratory plasma, Ed. E. W. Hones Jr., pp. 4-14, Geo- physical Monograph 30, AGU, Washington D.C., 1984.

Berger, M. A., Rigorous new limits on magnetic helicity dissipation in the solar corona, Geophys. Astrophys. Fluid Dynamics, 30, 79, 1984.

Birn, J., Computer studies of the dynamic evolution of the geomagnetic tail, J. Geophys. Res. 85, 1214, 1980.

Biskamp, D. and Welter, H., Coalescence of magnetic islands, Phys.Rev. Letters, 44, 1069-1072, 1980.

Büchner, J. and Kuska, J.-P., Three-dimensional collisionless reconnection through thin current sheets: Theory and selfconsistent simulations in Third International Conference on Substorms (ICS-3), ESA SP-389, 373, 1996.

Büchner, J. and Kuska, J.-P., Sausage mode instability of thin current sheets as a cause of magnetospheric substorms, Ann. Geophysicae, 17, 604-612, 1999.

Büchner, J., Three-dimensional magnetic reconnection in astrophysical plasmas - Kinetic approach, Astrophys. and Space Sci., 264, No. 1-4, 25-42, 1999.

Faires, J. D. and Burden, R. L., Numerical Methods, PWSPublishing Company, Boston, 1993.

Harris, E. G., On a plasma sheath separating regions of oppositely directed magnetic field, Nuovo Cimento, 23, 115, 1962.

Hesse, M. and Schindler, K., A theoretical foundation of general magnetic reconnection, J. Geophys. Res., 93 (A6), 5559, 1988.

Hornig, G. and Rastätter, L., The role of helicity in the reconnection process, Adv. Space Res, 19, 1789, 1997.

Hornig, G., Topological constraints on the relaxation of complex magnetic fields, 9-th European Meeting on Solar Physics: Magnetic fields and solar processes, Firenze, Italy, ESA SP-448, 605, 1999.

Hornig, G., The evolution of magnetic helicity under reconnection, in Magnetic Helicity in Space and Laboratory Plasmas, M. R. Brown, R. C. Canfield and A. A. Pevtsov (Eds.), Geophysical Monographs Vol. 111, American Geophysical Union, Washington, 157-165, 1999b.

Kuska, J. P. and Büchner, J., The three-dimensional fully kinetic electromagnetic PIC simulation code GISMO, in Proc. VIIth International Conference on Plasma Astrophysics and Space Physics, Kluwer, 645-52, 1999.

Levy, Y., Degani, P., and Seginer, A., Graphical visualization of vortical flows by means of helicity, AIAA, J. 28, 1347-1352, 1990.

Low, B. C., Magnetohydrodynamic processes in the solar corona: flares, coronal mass ejections, and magnetic helicity, Phys. Plasmas, 1, 1684-1690, 1994.

Matthaeus, W. H. and Lamkin, S. L., Rapid magnetic reconnection caused by finite amplitude fluctuations, Phys. Fluids, 28, 303307, 1985.

Matthaeus, W. H. and Lamkin, S. L., Turbulent magnetic reconnection, Phys. Fluids, 29, 2513-2534, 1986.

Moffat, H. K. and Tsinober, A., Helicity in laminar and turbulent flow, Ann. Rev. Fluid Mech., 24, 281-312, 1992.

Parker, E., Spontaneous Current Sheets in Magnetic Fields, Oxford Univ. Press, New York, 1994.

Potter, D., Computational Physics, John Wiley \& Sons Ltd., 1973.

Pritchett, P. L., Coroniti, F. V., and Decyk, V. K., Three-dimensional stability of thin quasi-neutral current sheets, J. Geophys. Res., 101, 27413, 1996.

Schindler, K., in B. M. McCormac (Ed.), Earth's Magnetospheric Processes, Reidel, Dordrecht, 200, 1972.

Schindler, K., Hesse, M., and Birn, J., General magnetic reconnection, parallel electric fields and helicity, J. Geophys. Res., 93 
(A6), 5547, 1988.

Schindler, K. and J. Birn, On the cause of thin current sheets in the near-Earth magnetotail and their possible significance for magnetospheric substorms, J. Geophys. Res., 98, 15477, 1993.

Schwenn, R., Inhester, B., Plunkett, S. P., Epple, A., Podlipnik, B., et al., First view of the extended green-line emission corona at solar activity minimum using the Lasco-C1 coronagraph on Soho, Solar Phys., 175, 667, 1997.

Taylor, J. B., Relaxation of toroidal plasma and generation of reverse magnetic fields, Physical Review Letters, 33 (19), 1139, 1974.

Terasawa, T., Hall current effect on tearing mode instabilities, J. Geophys. Res. 19, 475, 1983.

Vasyliūnas, V. M., Theoretical models of magnetic field line merging, Rev. Geophys. Space Phys., 13, 303, 1975.

Wiegelmann, T. and Schindler, K., Formation of thin current sheets in a quasistatic magnetotail model, Geophys. Res. Lett., 22, 2057, 1995.
Wiegelmann, T., Schindler, K., and Neukirch, T.: Helmet Streamers with Triple Structures: Simulations of resistive dynamics, Solar Phys., 191, 391, 2000.

Wiegelmann, T. and Büchner, J., Kinetic simulations of the coupling between current instabilities and reconnection in thin current sheets, Nonlinear Processes in Geophysics, 7, 141-150, 2000.

Winglee, R. M., Characteristics of the fields and particle acceleration during rapidly induced tail thinning and reconnection, in Magnetospheric Substorms, Eds. J. R. Kan, T. A. Potemra, S. Kokubun, and T. Iijima, Geophys. Monogr. Ser., vol. 64, p. 425, AGU, Washington D.C., 1991.

Woltjer, L., On the theory of hydromagnetic equilibrium, Rev. Mod. Phys, 32, 914-915, 1960.

Zhu, Z. W. and Winglee, R. M., Tearing instability, flux ropes, and the kinetic current sheet kink instability in the Earth's magnetotail: A 3-dimensional perspective from particle simulations, J. Geophys., 101, 4885-4897, 1996. 